\title{
Certain Subclass of Meromorphic Functions with Positive Coefficients Defined by Bessel Function
}

\author{
Santosh M. Popade ${ }^{1}$, Rajkumar N. Ingle ${ }^{2}$, P. Thirupathi Reddy ${ }^{3}$ and B. Venkateswarlu ${ }^{4 *}$
}

\begin{abstract}
The aim of the present paper is to introduce a class $\Sigma_{p}^{*}(G, H, \tau, c)$ of meromorphic univalent functions in $E=\{0<$ $|z|<1\}$ and investigate coefficient estimates, distortion properties and radius of convexity estimates for this class. Furthermore, it is shown that this class is closed under convex linear combinations, convolutions and integral transforms.
\end{abstract}

Keywords: Convolutions, distortion, meromorphic.

2010 AMS: 30C45

\section{${ }^{1}$ Department of Mathematics, Sant Tukaram College of Arts \& Science, Parbhani - 431 401, Maharastra, India.}

2 Department of Mathematics, Bahirji Smarak Mahavidyalay, Bashmathnagar - 431 512, Hingoli Dist., Maharastra, India.

${ }^{3}$ Department of Mathematics, Kakatiya University, Warangal- 506 009, Telangana, India, ORCID: 0000-0002-0034-444X

${ }^{4}$ Department of Mathematics, GSS, GITAM University, Doddaballapur- 561 163, Bengaluru Rural, India, ORCID: 0000-0003-3669-350X

*Corresponding author: bvlmaths@gmail.com

Received: 22 April 2020, Accepted: 1 July 2020, Available online: 29 September 2020

\section{Introduction}

Let $\Sigma$ symbolized the class of analytic functions, which are with a simple pole at the origin with residue 1 of the form in the punctured unit disc $E=\{z: 0<|z|<1\}$ and of the form

$$
f(z)=\frac{1}{z}+\sum_{n=1}^{\infty} a_{n} z^{n}
$$

Let $\Sigma_{s}, \Sigma^{*}(\alpha)$ and $\Sigma_{k}(\alpha)$ be the subclass of $\Sigma$ consisting of univalent, meromorphically starlike of order $\alpha$ and meromorphically convex functions of $\alpha, 0 \leq \alpha<1$ respectively.

A function given by (1.1) is in the $\Sigma^{*}(\alpha)$

$$
\Leftrightarrow \Re\left\{-\frac{z f^{\prime}(z)}{f(z)}\right\}>\alpha(z \in E)
$$

and $f \in \Sigma_{k}(\alpha)$

$$
\Leftrightarrow \Re\left\{-\frac{\left(1+z f^{\prime \prime}(z)\right)}{f^{\prime}(z)}\right\}>\alpha,(z \in E) .
$$

Recent years, many authors investigated the subcalss of meromorphic functions with positive coefficients (see $[1,2,3,4,5]$. 
Let $\Sigma_{p}$ denote the class of functions of the form

$$
f(z)=\frac{1}{z}+\sum_{n=1}^{\infty} a_{n} z^{n},\left(a_{n} \geq 0\right)
$$

that are analytic and univalent in $E$.

We recall here the generalized Bessel function of first kind of order $\gamma$ (see [6]), denoted by

$$
w(z)=\sum_{n=0}^{\infty} \frac{(-c)^{n}}{n ! \Gamma\left(\gamma+n+\frac{b+1}{2}\right)}\left(\frac{z}{2}\right)^{2 n+\gamma}(z \in U)
$$

(where $\Gamma$ stands for the Gamma Euler function) which is the particular solution of the second order linear homogeneous differential equation (see, for details, [7] )

$$
z^{2} w^{\prime \prime}(z)+b z w^{\prime}(z)+\left[c z^{2}-\gamma^{2}+(1-b) \gamma\right] w(z)=0,
$$

where $c, \gamma, b \in C$.

We introduce the function $\varphi$ defined, in terms of the generalized Bessel function $w$ by

$$
\varphi(z)=2^{\gamma} \Gamma\left(\gamma+\frac{b+1}{2}\right) z^{-\left(1+\frac{\gamma}{2}\right)} w(\sqrt{z}) .
$$

By using the well-known Pochhammer symbol $(x)_{\tau}$ defined, for $x \in C$ and in terms of the Euler gamma function, by

$$
(x)_{\tau}=\frac{\Gamma(x+\tau)}{\Gamma(x)}= \begin{cases}1, & (\tau=0) \\ x(x+1)(x+2) \cdots(x+n-1), & (\tau=n \in N=\{1,2,3 \cdots\}) .\end{cases}
$$

We obtain the following series representation for the function $\varphi(z)$

$$
\varphi(z)=\frac{1}{z}+\sum_{n=0}^{\infty} \frac{(-c)^{n+1}}{4^{n+1}(n+1) !(\tau)_{n+1}} z^{n}\left(\tau=\gamma+\frac{b+1}{2} \notin Z_{0}^{-}=\{0,-1,-2, \cdots\}\right) .
$$

Corresponding to the function $\varphi$ define the Bessel operator $S_{\tau}^{c}$ by the following Hadamard product

$$
\begin{aligned}
S_{\tau}^{c} f(z)=(\varphi * f)(z) & =\frac{1}{z}+\sum_{n=0}^{\infty} \frac{\left(\frac{-c}{4}\right)^{n+1} a_{n}}{(n+1) !(\tau)_{n+1}} z^{n} \\
& =\frac{1}{z}+\sum_{n=1}^{\infty} \phi(n, \tau, c) a_{n} z^{n},
\end{aligned}
$$

where $\phi(n, \tau, c)=\frac{\left(\frac{-c}{4}\right)^{n}}{(n) !(\tau)_{n}}$

Definition 1.1. Let $\Sigma_{p}^{*}(G, H, \tau, c)$ denote the subclass of $\Sigma_{p}$ consisting of functions $f(z)$ in $\Sigma_{p}$ which satisfy

$$
\left|\frac{z\left(S_{\tau}^{c} f(z)\right)^{\prime}}{S_{\tau}^{c} f(z)}+1\right|<\left|G+H \frac{z\left(S_{\tau}^{c} f(z)\right)^{\prime}}{S_{\tau}^{c} f(z)}\right|,
$$

for $-1 \leq G<H, 0<H \leq 1$.

\section{Coefficient Inequalities}

Our first theorem gives a necessary and sufficient condition for a function to be in $\Sigma_{p}^{*}(G, H, \tau, c)$.

Theorem 2.1. Let $f(z) \in \Sigma_{p}$ as given by (1.4). Then $f(z) \in \Sigma_{p}^{*}(G, H, \tau, c)$ if and only if

$$
\sum_{n=1}^{\infty}[(n+1)+(G+H n)] \phi(n, \tau, c) a_{n} \leq H-G,
$$

for $-1 \leq G<H, 0<H \leq 1$. 
Proof. Suppose $f(z)=\frac{1}{z}+\sum_{n=1}^{\infty} a_{n} z^{n}, a_{n} \geq 0$, is in $\Sigma_{p}^{*}(G, H, \tau, c)$. Then

$$
\left|\frac{\frac{z\left(S_{\tau}^{c} f(z)\right)^{\prime}}{S_{\tau}^{c} f(z)}+1}{G+H \frac{z\left(S_{\tau}^{c} f(z)\right)^{\prime}}{S_{\tau}^{c} f(z)}}\right|=\left|\frac{\sum_{n=1}^{\infty}(n+1) \phi(n, \tau, c) a_{n} z^{n}}{(H-G) \frac{1}{z}-\sum_{n=1}^{\infty}(G+H n) \phi(n, \tau, c) a_{n} z^{n}}\right|<1
$$

for all $z \in E$. Since $\operatorname{Re}(z) \leq|z|$ for all $z$, we have

$$
\operatorname{Re}\left\{\frac{\sum_{n=1}^{\infty}(n+1) \phi(n, \tau, c) a_{n} z^{n}}{(H-G) \frac{1}{z}-\sum_{n=1}^{\infty}(G+H n) \phi(n, \tau, c) a_{n} z^{n}}\right\}<1,(z \in E) .
$$

Now choose the values of $z$ on real axis so that $\frac{z\left(S_{\tau}^{c} f(z)\right)^{\prime}}{S_{\tau}^{c} f(z)}$ is real.

Upon clearing the denominator in (2.2) and letting $z \rightarrow 1$ through positive values, we obtain

$$
\sum_{n=1}^{\infty}[(n+1)+(G+H n)] \phi(n, \tau, c) a_{n} \leq H-G .
$$

Conversely, suppose that (2.1) holds for all admissible values of $G$ and $H$. We have

$$
\begin{aligned}
M\left(f, f^{\prime}\right) & =\left|z\left(S_{\tau}^{c} f(z)\right)^{\prime}+S_{\tau}^{c} f(z)\right|-\left|G S_{\tau}^{c} f(z)+H z\left(S_{\tau}^{c} f(z)\right)^{\prime}\right| \\
& =\left|\sum_{n=1}^{\infty}(n+1) \phi(n, \tau, c) a_{n} z^{n}\right|-\left|(H-G) \frac{1}{z}-\sum_{n=1}^{\infty}(G+H n) \phi(n, \tau, c) a_{n} z^{n}\right| \\
\text { or } & \\
z M\left(f, f^{\prime}\right) & \leq \sum_{n=1}^{\infty}(n+1) \phi(n, \tau, c) a_{n}|z|^{n+1}-(H-G)+\sum_{n=1}^{\infty}(G+H n) \phi(n, \tau, c) a_{n}|z|^{n+1} \\
& =\sum_{n=1}^{\infty}[(n+1)+(G+H n)] \phi(n, \tau, c) a_{n}|z|^{n+1}-(H-G) .
\end{aligned}
$$

Since the above inequality holds for all $r=|z|, 0<r<1$, letting $r \rightarrow 1$, we have

$$
\sum_{n=1}^{\infty}[(n+1)+(G+H n)] \phi(n, \tau, c) a_{n} \leq(H-G)
$$

by (2.1). Hence it follows that $f(z)$ is in the class $\Sigma_{p}^{*}(G, H, \tau, c)$.

Corollary 2.2. If the function $f(z) \in \Sigma_{p}^{*}(G, H, \tau, c)$ then

$$
a_{n} \leq \frac{(H-G)}{[(n+1)+(G+H n)] \phi(n, \tau, c)},(n \geq 1) .
$$

The result is sharp for the function

$$
f_{n}(z)=\frac{1}{z}+\frac{(H-G)}{[(n+1)+(G+H n)] \phi(n, \tau, c)} z^{n},(n \geq 1) .
$$

\section{Distortion Properties and Radius of Convexity Estimates}

Theorem 3.1. If the function $f(z) \in \Sigma_{p}^{*}(G, H, \tau, c)$ then for $0 \leq|z|=r<1$,

$$
\frac{1}{r}-\frac{(H-G)}{(2+G+H) \phi(1, \tau, c)} r \leq|f(z)| \leq \frac{1}{r}+\frac{(H-G)}{(2+G+H) \phi(1, \tau, c)} r .
$$

The result is sharp. 
Proof. Suppose $f(z)$ is in $\Sigma_{p}^{*}(G, H, \tau, c)$. By Theorem 2.1, we have

$$
\sum_{n=1}^{\infty} a_{n} \leq \frac{(H-G)}{[(n+1)+(G+H n)] \phi(n, \tau, c)}, \quad(n \geq 1) .
$$

Thus $|f(z)| \leq \frac{1}{|z|}+|z| \sum_{n=1}^{\infty} a_{n} \leq \frac{1}{r}+\frac{(H-G)}{(2+G+H) \phi(1, \tau, c)} r$.

Also $|f(z)| \geq \frac{1}{|z|}-|z| \sum_{n=1}^{\infty} a_{n} \geq \frac{1}{r}-\frac{(H-G)}{(2+G+H) \phi(1, \tau, c)} r$.

Thus the result is sharp for the function

$$
f(z)=\frac{1}{z}+\frac{(H-G)}{(2+G+H) \phi(1, \tau, c)} z .
$$

Theorem 3.2. If the function $f(z) \in \Sigma_{p}^{*}(G, H, \tau, c)$ then $f(z)$ is meromorphically convex of order $\delta(0 \leq \delta<1)$ in $|z|<r=$ $r(G, H, \tau, c, \delta)$, where

$$
r(G, H, \tau, c, \delta)=\inf _{n \geq 1}\left[\frac{(1-\delta)[(n+1)+(G+H n)] \phi(n, \tau, c)}{(H-G) n(n+2-\delta)}\right]^{\frac{1}{n+1}} .
$$

The result is sharp.

Proof. Let $f(z)$ be in $\Sigma_{p}^{*}(G, H, \tau, c)$. Then by Theorem 2.1, we have

$$
\sum_{n=1}^{\infty} \frac{[(n+1)+(G+H n)] \phi(n, \tau, c)}{(H-G)} a_{n} \leq 1
$$

It is sufficient to show that $\left|2+\frac{z f^{\prime \prime}(z)}{f^{\prime}(z)}\right| \leq 1-\delta$ for $|z| \leq r(G, H, \tau, c, \delta)$, where $r(G, H, \tau, c, \delta)$ is as specified in the statement of the theorem. Then

$$
\left|2+\frac{z f^{\prime \prime}(z)}{f^{\prime}(z)}\right|=\left|\frac{\sum_{n=1}^{\infty} n(n+1) a_{n} z^{n-1}}{\frac{-1}{z^{2}}+\sum_{n=1}^{\infty} n a_{n} z^{n-1}}\right| \leq \frac{\sum_{n=1}^{\infty} n(n+1) a_{n}|z|^{n+1}}{1-\sum_{n=1}^{\infty} n a_{n}|z|^{n+1}} .
$$

This will be bounded by $1-\delta$ if

$$
\sum_{n=1}^{\infty} \frac{n(n+2-\delta)}{1-\delta} a_{n}|z|^{n+1} \leq 1
$$

By (3.3), it follows that (3.4) is true if

$$
\frac{n(n+2-\delta)}{1-\delta}|z|^{n+1} \leq \frac{[(n+1)+(G+H n)] \phi(n, \tau, c)}{H-G},(n \geq 1)
$$

or

$$
|z| \leq\left\{\frac{(1-\delta)[(n+1)+(G+H n)] \phi(n, \tau, c)}{(H-G) n(n+2-\delta)}\right\}^{\frac{1}{n+1}},(n \geq 1) .
$$

Setting $|z|=r(G, H, \tau, c, \delta)$ in (3.5), the result follows.

The result is sharp for the functions

$$
f_{n}(z)=\frac{1}{z}+\frac{(H-G)}{[(n+1)+(G+H n)] \phi(n, \tau, c)} z^{n},(n \geq 1) .
$$




\section{Convex Linear Combinations and Convolution Properties}

We shall prove that the class $\Sigma_{p}^{*}(G, H, \tau, c)$ is closed under convex linear combinations and convolutions.

Theorem 4.1. Let $f_{0}(z)=\frac{1}{z}$ and

$$
f_{n}(z)=\frac{1}{z}+\frac{(H-G)}{[(n+1)+(G+H n)] \phi(n, \tau, c)} z^{n},(n \geq 1) .
$$

Then $f(z) \in \Sigma_{p}^{*}(G, H, \tau, c)$ if and only if it can be expressed in the form $f(z)=\sum_{n=0}^{\infty} \lambda_{n} f_{n}(z)$, where $\lambda_{n} \geq 0$ and $\sum_{n=0}^{\infty} \lambda_{n}=1$.

Proof. Let $f(z)=\sum_{n=0}^{\infty} \lambda_{n} f_{n}(z)$ with $\lambda_{n} \geq 0$ and $\sum_{n=0}^{\infty} \lambda_{n}=1$. Then

$$
f(z)=\sum_{n=0}^{\infty} \lambda_{n} f_{n}(z)=\frac{1}{z}+\sum_{n=0}^{\infty} \frac{(H-G)}{[(n+1)+(G+H n)] \phi(n, \tau, c)} z^{n} .
$$

Since

$$
\begin{aligned}
& \frac{[(n+1)+(G+H n)] \phi(n, \tau, c)}{(H-G)} \lambda_{n} \frac{(H-G)}{[(n+1)+(G+H n)] \phi(n, \tau, c)} \\
= & \sum_{n=1}^{\infty} \lambda_{n}=1-\lambda_{n} \leq 1,
\end{aligned}
$$

by Theorem 2.1, $f(z)$ is in the class $\Sigma_{p}^{*}(G, H, \tau, c)$.

Conversely, suppose that the function $f(z)$ is the class $\Sigma_{p}^{*}(G, H, \tau, c)$. Setting

$$
\lambda_{n}=\frac{[(n+1)+(G+H n)] \phi(n, \tau, c)}{(H-G)} a_{n}, n \geq 1
$$

and $\lambda_{0}=1-\sum_{n=1}^{\infty} \lambda_{n}$, it follows that $f(z)=\sum_{n=0}^{\infty} \lambda_{n} f_{n}(z)$.

Here, we see that $\lambda_{n} \geq 0(n \geq 1)$ by definition and $\lambda_{0} \geq 0$ in view of Theorem 2.1. This completes the proof of the theorem.

The result is sharp for the function

$$
f_{n}(z)=\frac{1}{z}+\frac{(H-G)}{[(n+1)+(G+H n)] \phi(n, \tau, c)} z^{n},(n \geq 1) .
$$

Robertson [8], has shown that if $f(z)=\frac{1}{z}+\sum_{n=1}^{\infty} a_{n} b_{n} z^{n}$ and $g(z)=\frac{1}{z}+\sum_{n=1}^{\infty} b_{n} z^{n}$ are in $\Sigma_{s}$ then so their convolutions $(f * g)(z)=\frac{1}{z}+\sum_{n=1}^{\infty} a_{n} b_{n} z^{n}$.

Theorem 4.2. If the function $f(z)$ and $g(z)$ are in the class $\Sigma_{p}^{*}(G, H, \tau, c)$ then $(f * g)(z)$ is the class $\Sigma_{p}^{*}(G, H, \tau, c)$.

Proof. Suppose that $f(z)$ and $g(z)$ are in $\Sigma_{p}^{*}(G, H, \tau, c)$. By Theorem 2.1, we have

$$
\begin{aligned}
& \sum_{n=1}^{\infty} \frac{[(n+1)+(G+H n)] \phi(n, \tau, c)}{(H-G)} a_{n} \leq 1, \\
& \sum_{n=1}^{\infty} \frac{[(n+1)+(G+H n)] \phi(n, \tau, c)}{(H-G)} b_{n} \leq 1 .
\end{aligned}
$$

Since $f(z)$ and $g(z)$ are regular in $E$, so $(f * g)(z)$. Furthermore

$$
\begin{aligned}
& \sum_{n=1}^{\infty} \frac{[(n+1)+(G+H n)] \phi(n, \tau, c)}{(H-G)} a_{n} b_{n} \leq\left\{\frac{[(n+1)+(G+H n)] \phi(n, \tau, c)}{(H-G)}\right\}^{2} a_{n} b_{n} \\
& \leq \sum_{n=1}^{\infty} \frac{[(n+1)+(G+H n)] \phi(n, \tau, c)}{(H-G)} a_{n}\left\{\frac{[(n+1)+(G+H n)] \phi(n, \tau, c)}{(H-G)}\right\} b_{n} \\
& \leq 1 .
\end{aligned}
$$

Hence by Theorem 2.1, $(f * g)(z)$ is in the class $\Sigma_{p}^{*}(G, H, \tau, c)$. 


\section{Integral Transforms}

In this section, we consider transforms of functions in the class $\Sigma_{p}^{*}(G, H, \tau, c)$ of the type considered by Bajpai [9].

Theorem 5.1. If the function $f(z)$ is in the class $\Sigma_{p}^{*}(G, H, \tau, c)$ then the integral transforms

$$
F_{c}(z)=c \int_{0}^{1} u^{c} f(u z) d z,(0<c<\infty)
$$

is in the class $\Sigma_{p}^{*}(G, H, \tau, c)$.

Proof. Suppose $f(z)$ is in $\Sigma_{p}^{*}(G, H, \tau, c)$. Then we have

$$
F_{c}(z)=c \int_{0}^{1} u^{c} f(u z) d u=\frac{1}{z}+\sum_{n=1}^{\infty} \frac{c}{n+c+1} a_{n} z^{n} .
$$

Since

$$
\sum_{n=1}^{\infty} \frac{[(n+1)+(G+H n)] \phi(n, \tau, c)}{(H-G)} \frac{c a_{n}}{n+c+1} \leq \frac{\sum_{n=1}^{\infty}[(n+1)+(G+H n)] \phi(n, \tau, c)}{(H-G)} a_{n} \leq 1,
$$

by Theorem 2.1, it follows that $F_{c}(z)$ is in the class $\Sigma_{p}^{*}(G, H, \tau, c)$.

\section{Acknowledgement}

I warmly thank the referees for the careful reading of the paper and their comments.

\section{References}

[1] J. Clunie, On meromorphic schlicht functions, J. Londan Math. Soc., 34 (1959), 215-216 .

[2] J. E. Miller, Convex meromorphic mapping and related functions, Proc. Amer. Math. Soc., 25 (1970), $220-228$.

[3] Ch. Pommarenke, On meromorphic starlike functions, Pecific , J. Math., 13 (1963), 221-235 .

[4] B.A. Uralegaddi and M. D. Ganigi, A certain class of meromorphic celly starlike functions with positive coefficients, Pure. Appl. Math. Sci., 26 ( 1987), 75-81.

[5] B. Venkateswarlu, P. Thirupathi Reddy, Ch. Meng and R. Madhuri Shilpa, A new subclass of meromorphic functions with positive coefficients defined by Bessel function, Note di Matematica (Accepted).

[6] E. Deniz, H. Orhan and H.M. Srivastava, Some sufficient conditions for univalence of certain families of integral operators involving generalized Bessel functions, Taiwan J. Math., 15(2) (2011), 883-917 .

[7] G. N. Watson, A treatise on the theory of Bessel functions, 2nd edn. Cambridge University Press, Cambridge, (1994).

[8] M. S. Robertson, Convolution of Schlicht functions, Proc. Amer. Math. Soc., 13 (1962), 585-587.

[9] S. K. Bajpai, A note on a class of meromorphic univalent functions, Rev. Roumanie Math. Pure Appl., 22 (1997) , $295-297$. 